\title{
The Impact of Immunosuppression and Autoimmune Disease on Severe Outcomes in Patients Hospitalized with COVID-19
}

\author{
Enric Monreal ${ }^{1}$ (1) Susana Sainz de la Maza ${ }^{1}$ - Jose Ignacio Fernández-Velasco ${ }^{2}$ - Elena Natera-Villalba ${ }^{1}$. \\ Claudia Geraldine Rita ${ }^{2}$ - Fernando Rodríguez-Jorge ${ }^{1}$ - Álvaro Beltrán-Corbellini ${ }^{1}$ - Ignacio Iturrieta-Zuazo ${ }^{2}$. \\ Enrique Rodríguez de Santiago ${ }^{3}$. Mercedes Espiño ${ }^{2}$. Ana de Andrés ${ }^{2}$. Jesús Fortún ${ }^{4}$. Esther Barbero ${ }^{5}$. \\ Mónica Vázquez $^{6}$ - Milagros Fernández Lucas $^{7} \cdot$ Luis Manzano $^{8}$. Beatriz Montero-Errasquín ${ }^{9}$. \\ Lucienne Costa-Frossard ${ }^{1}$. Jaime Masjuan ${ }^{1} \cdot$ Luisa María Villar $^{2}$ • for the COVID-HRC group
}

Received: 8 September 2020 / Accepted: 16 November 2020 / Published online: 24 November 2020

(C) Springer Science+Business Media, LLC, part of Springer Nature 2020

\begin{abstract}
Immunosuppression (IS) and autoimmune disease (AD) are prevalent in patients with severe coronavirus disease 2019 (COVID-19), but their impact on its clinical course is unknown. We investigated relationships between IS, AD, and outcomes in patients hospitalized with COVID-19. Data on consecutive admissions for COVID-19 were extracted retrospectively from medical records. Patients were assigned to one of four cohorts, according to whether or not they had an $\mathrm{AD}$ (AD and NAD) or were immunosuppressed (IS and NIS). The primary endpoint was development of severe acute respiratory distress syndrome (ARDS); secondary endpoints included death, and a composite of mechanical ventilation (MV) or death. A total of 789 patients were included: $569(72.1 \%)$ male, 76 (9.6\%) with an $\mathrm{AD}$, and $63(8.0 \%)$ with IS. Relative to the NIS-NAD cohort, patients in the IS-AD cohort had a significantly reduced risk of severe ARDS (adjusted hazard ratio [aHR] 0.42;95\% confidence interval [CI] $0.23-0.80 ; p=0.008$ ). No significant relationships between IS or AD status and either death or the composite of MV and death were identified, although a trend towards higher mortality was identified in the IS-NAD cohort (aHR vs NIS-NAD 1.71; 95\% CI $0.94-3.12 ; p=0.081$ ). Patients in this cohort also had higher median serum levels of interleukin-6 compared with IS-AD patients $(98.2$ vs $21.6 \mathrm{pg} / \mathrm{mL} ; p=0.0328)$ and NIS-NAD patients $(29.1 \mathrm{pg} / \mathrm{mL} ; p=0.0057)$. In conclusion, among patients hospitalized with COVID-19, those receiving immunosuppressive treatment for an AD may have a reduced risk of developing severe ARDS.
\end{abstract}

Keywords COVID-19 - severe acute respiratory syndrome coronavirus $2 \cdot$ autoimmune diseases $\cdot$ immunosuppression

Enric Monreal

enrique.monreal@salud.madrid.org

1 Department of Neurology, Hospital Universitario Ramón y Cajal, Universidad de Alcalá, IRYCIS, Crta Colmenar Viejo, km 9,100, 28034 Madrid, Spain

2 Department of Immunology, Hospital Universitario Ramón y Cajal, Universidad de Alcalá, IRYCIS, Madrid, Spain

3 Department of Gastroenterology and Hepatology, Hospital Universitario Ramón y Cajal, Universidad de Alcalá, IRYCIS, Madrid, Spain

4 Department of Infectious Diseases, Hospital Universitario Ramón y Cajal, Universidad de Alcalá, IRYCIS, Madrid, Spain
5 Department of Pneumology, Hospital Universitario Ramón y Cajal, Universidad de Alcalá, IRYCIS, Madrid, Spain

6 Department of Rheumatology, Hospital Universitario Ramón y Cajal, Universidad de Alcalá, IRYCIS, Madrid, Spain

7 Department of Nephrology, Hospital Universitario Ramón y Cajal, Universidad de Alcalá, IRYCIS, Madrid, Spain

8 Department of Internal Medicine, Hospital Universitario Ramón y Cajal, Universidad de Alcalá, IRYCIS, Madrid, Spain

9 Department of Geriatrics, Hospital Universitario Ramón y Cajal, Universidad de Alcalá, IRYCIS, Madrid, Spain 


\section{Introduction}

Coronavirus disease 2019 (COVID-19), the clinical illness caused by severe acute respiratory syndrome coronavirus 2 (SARS-CoV-2), was first reported in Wuhan, China, on 31 December 2019 [1], and has since spread rapidly across the world. The first published data from China suggested that nearly one-third of patients may go on to develop acute respiratory distress syndrome (ARDS), a complication associated with increased need for mechanical ventilation (MV) and risk of death [2]. Older age and the presence of comorbidities were identified early on as being risk factors for ARDS, and for progression from ARDS to death [3].

Patients with immunosuppression were initially thought to be at increased risk of severe COVID-19. However, preliminary data have suggested that the immune response to SARSCoV-2 has two distinct phases [4]. The first (viral response) phase is triggered by the virus itself, and is characterized by mild constitutional symptoms. The second phase consists of an unregulated, excessive inflammatory response, which appears to be the main driver of lung tissue damage in COVID19 [5]. There would therefore appear to be a strong scientific rationale for therapeutic immunomodulation in mid- to latestage infection with SARS-CoV-2.

In a recent retrospective pilot study [6], we found that nonseverely immunosuppressed individuals admitted to our institution with confirmed COVID-19 were significantly less likely to develop moderate or severe ARDS than were nonimmunosuppressed individuals (adjusted odds ratio [OR] $0.16 ; p=0.004)$. After stratifying the immunosuppressed (IS) cohort by the presence or absence of an autoimmune disease (AD), a significantly lower risk was observed exclusively among IS-AD patients (adjusted OR 0.12; $p=0.007$ ). Thus, IS patients without an AD may have a more intense inflammatory response to infection with SARS-CoV-2 relative to those with an AD.

Based on these observations, we wanted to investigate whether patients with an $\mathrm{AD}$ might have a lower risk of having a severe outcome related to COVID-19 in comparison to those without an $\mathrm{AD}$. We also sought to determine whether IS patients without an $\mathrm{AD}$ (e.g., those receiving cancer chemotherapy) may have worse outcomes compared with other groups.

To test these hypotheses, we retrospectively analyzed available data from a large cohort of patients consecutively admitted to hospital with symptoms of COVID-19.

\section{Methods}

\section{Study Design}

This single-center, retrospective, observational study was performed at Hospital Universitario Ramón y Cajal (HRC) in
Madrid. Electronic medical records for all adults admitted to $\mathrm{HRC}$ with confirmed or highly suspected SARS-CoV-2 infection, from the beginning of the pandemic up to and including 15 April 2020, were retrieved for analysis.

Patients were included if they had a positive, laboratoryconfirmed test for SARS-CoV-2, and a predefined minimum of available baseline and follow-up data, including cytokine levels.

The study was approved by the institutional ethics board of HRC. Due to its retrospective design, the need for informed consent from individual patients was waived.

\section{Data Collection}

Trained physicians extracted data from medical records for the period between admission and discharge, death or 19 April 2020, whichever occurred first.

We recorded demographic information, medical history, comorbidities, hematologic and other laboratory data, radiologic findings, and clinical and respiratory variables; details of treatments administered for COVID-19 were also recorded. Patients were first stratified according to whether or not they had a diagnosed $\mathrm{AD}$, and then according to whether they were taking any drug with an immunomodulatory or immunosuppressive effect. ${ }^{1}$ Thus, the four cohorts of interest in the study were:

- IS-AD: IS patients with an AD (e.g., multiple sclerosis [MS] treated with fingolimod);

- NIS-AD: non-IS patients with an AD;

- IS-NAD: IS patients with no AD (e.g., a patient receiving calcineurin inhibitor therapy after kidney transplantation);

- NIS-NAD: non-IS patients with no AD.

The primary endpoint was the development of severe ARDS, according to the Berlin Definition [7]. To provide a pathophysiologic explanation for our results, we compared cytokine levels between cohorts. The secondary endpoints were death, and the combined endpoint of death or need for ventilation. Patients requiring ventilation received either MV or non-invasive ventilation (NIV), as clinically indicated. For the purposes of analysis, we did not differentiate between these treatments.

\section{Cytokine Quantification}

Serum cytokines (interleukin [IL]-1 $\beta$, IL-6, IL-8, IL-10, IL$12 \mathrm{p} 70$, and tumor necrosis factor $[\mathrm{TNF}] \alpha$ ) were quantified using the BD Cytometric Bead Array Human Inflammatory

\footnotetext{
${ }^{1}$ Immunomodulation was defined as an immunologic mechanism of action other than cell depletion. Corticosteroids were considered immunomodulators at methylprednisolone-equivalent dosages $<1 \mathrm{mg} / \mathrm{kg} /$ day, but immunosuppressants (i.e., drugs causing cell depletion or function decrease) at $\geq 1 \mathrm{mg} / \mathrm{kg} /$ day.
} 
Cytokines Kit and analyzed with FCAO Array Software v3.0 (both BD Biosciences, Franklin Lakes, NJ, USA).

\section{Statistical Analysis}

Continuous variables were described using means and standard deviations, or medians and interquartile ranges (IQRs), depending on whether or not data were normally distributed. Categorical variables were described using absolute and relative frequencies.

The analysis of variance (ANOVA) or Kruskal-Wallis tests were used for continuous variables, whereas the chi-squared or Fisher's exact test was used to analyze categorical variables. We used both unadjusted (crude) and multivariate (adjusted) Cox proportional-hazards models to perform survival analyses of all outcomes, the adjusted analyses accounting for potential confounding factors identified in the literature (sex, obesity, and age-adjusted Charlson Comorbidity Index (CCI) score [8]). ${ }^{2}$ Hazard ratios (HRs) and 95\% confidence intervals $(\mathrm{CI})$ were calculated relative to the NIS-NAD cohort.

All analyses were conducted using Stata ${ }^{\circledR} 14$ (StataCorp, College Station, TX, USA) and were two-tailed, with $p<0.05$ as the level of significance.

\section{Results}

\section{Patient Characteristics}

Eight hundred and forty-one patients fulfilled the eligibility criteria and were screened for inclusion. Forty-eight patients with a negative RT-PCR test and 4 patients with incomplete follow-up data were excluded; the remaining 789 participants were included (Fig. 1).

At admission, 76 patients (9.6\%) had at least one AD; among them, 37 were immunosuppressed (IS-AD cohort), and 39 were not immunosuppressed (NIS-AD cohort).

Most patients in the study $(n=713 ; 90.4 \%)$ did not have an AD. However, 26 of these patients were immunosuppressed (the IS-NAD cohort). By far the largest cohort in the study was that of patients with neither an AD nor immunosuppression (NIS-NAD; $n=687$ ).

Sixty-three patients $(8.0 \%)$ were classed as immunosuppressed on admission. The most frequently used immunosuppressants were "classical" agents (e.g., mycophenolate mofetil and calcineurin inhibitors; these were used in $35.1 \%$ of IS-AD patients and $26.9 \%$ of IS-NAD patients) and cancer chemotherapy (which were used in $46.2 \%$ of IS-NAD patients). Full

\footnotetext{
2 The CCI is a weighted index that summarizes, as a single number, comorbidities that are associated with increased mortality or resource utilization. Its use allows multivariate models to be adjusted using a single (instead of multiple) covariate.
}

details of baseline treatments in the IS-AD and IS-NAD cohorts are shown in Supplementary Table S1 (Electronic Supplementary Material). Immunosuppressive treatment was more frequently discontinued at admission in IS-AD (21/37; $56.8 \%)$ compared with IS-NAD (11/26; $42.3 \%)$ patients.

The median (IQR) time from onset of symptoms to admission was 7 (5-10) days, and the median duration of hospitalization was 14 (10-21) days.

Baseline demographics, clinical data, and laboratory findings are shown in Table 1 . The median age of the study population was 64 years, and over $70 \%$ were male. There were significantly fewer men in the IS-AD cohort (45.9\%) than in either the IS-NAD $(76.9 \% ; p=0.02)$ or NIS-NAD $(74.1 \%$; $p<0.0001)$ cohorts. Relevant comorbidities were common in all cohorts, particularly hypertension and obesity; however, obesity was less prevalent in the IS-AD cohort than in the other cohorts. As expected, chronic renal disease, active malignancy, and organ transplantation were significantly more common in the IS-NAD cohort than in other cohorts.

The median (IQR) CCI score for the overall study population was $3(1-4)$. The highest and lowest values were in the IS-NAD (score 6) and NIS-NAD (2) cohorts, respectively; these scores were significantly different to the IS-AD cohort score (3).

Baseline blood gas and hematology data are shown in Supplementary Table S2 (Electronic Supplementary Material). Although median leukocyte and neutrophil counts were within normal ranges, they were lower in the IS-NAD vs other cohorts.

\section{Outcomes}

Severe ARDS developed in 452 (57.3\%) patients overall, and was less frequent among patients with an $\mathrm{AD}$ than in those without ( $43.4 \%$ vs $58.8 \%$ ). The overall cumulative incidence of severe ARDS was $18.2 \%$ at 7 days from symptom onset, and $63.4 \%$ at 28 days (Supplementary Table S3). After stratifying by $\mathrm{AD}$, cumulative incidence at 7 and 28 days was $15.8 \%$ and $43.5 \%$, respectively, in the $\mathrm{AD}$ cohort, and $18.5 \%$ and $65.8 \%$, respectively, in the NAD cohort (adjusted HR 0.69 ; 95\% CI 0.48-0.99; $p=0.044)$.

Immunosuppressed patients with an $\mathrm{AD}$ (i.e., IS-AD) had a significantly reduced risk of developing severe ADRS in comparison to patients with neither immunosuppression nor an AD (NIS-NAD), in both the unadjusted (HR 0.37; 95\% CI $0.19-0.68 ; p=0.002$ ) and adjusted (HR $0.42 ; 95 \%$ CI 0.23 $0.80 ; p=0.008$ ) analyses (Table 2 and Fig. 2). No such reduction was found in the NIS-AD cohort (adjusted HR 0.96; 95\% CI 0.63-1.47), while in the IS-NAD cohort there was a nonsignificant increase in risk relative to the NIS-NAD cohort (adjusted HR 1.14; 95\% CI 0.72-1.81). To investigate the robustness of these findings, we performed two sensitivity analyses: (i) using treatments administered during admission 


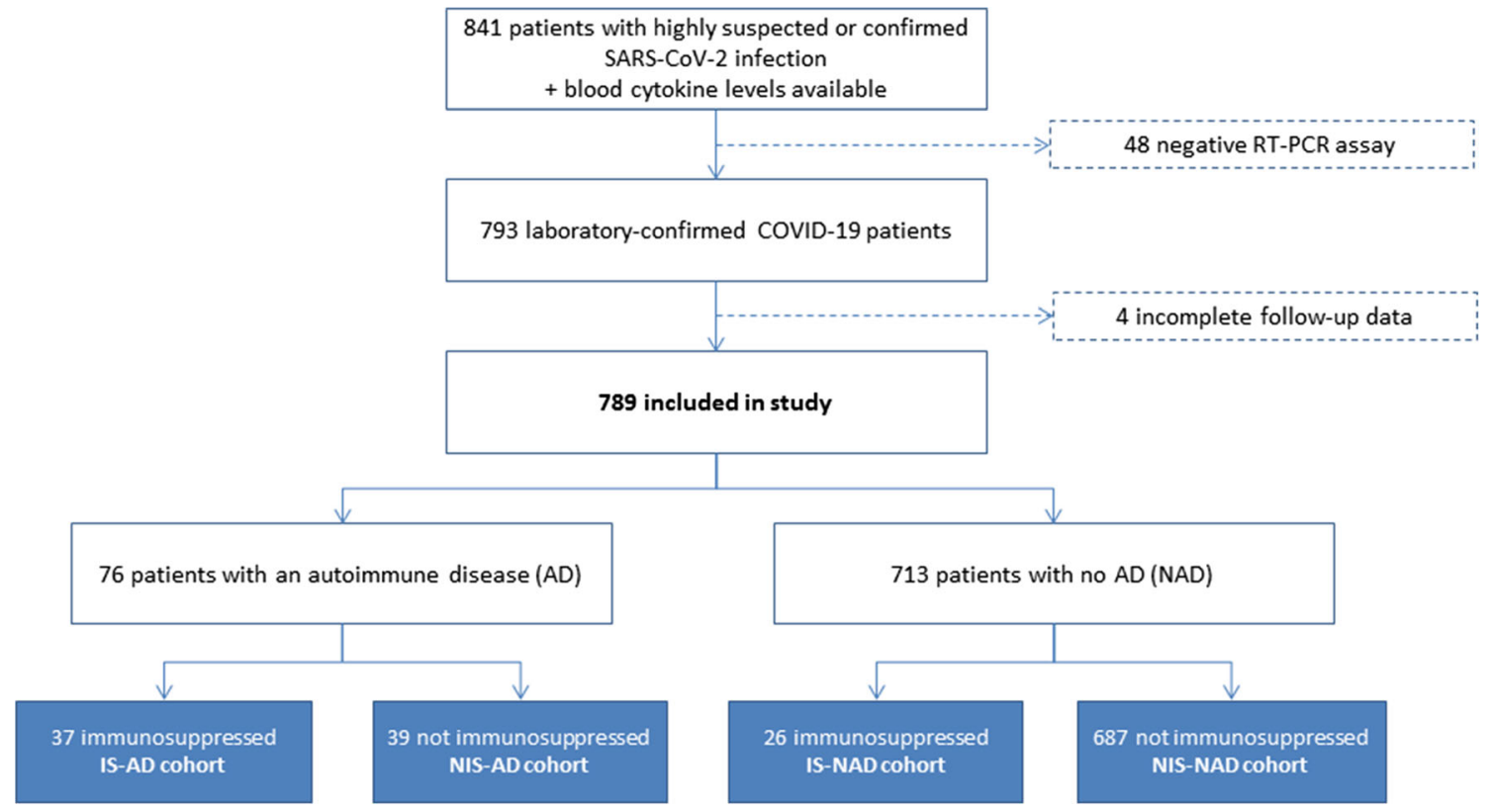

Fig. 1 Patient inclusion and stratification. COVID-19, coronavirus disease 2019; IS, immunosuppressed; NIS, not immunosuppressed; RT-PCR, reverse transcriptase-polymerase chain reaction; SARS-CoV-2, severe acute respiratory syndrome coronavirus 2

Table 1 Baseline demographics and clinical data

\begin{tabular}{|c|c|c|c|c|c|}
\hline \multirow[t]{2}{*}{ Parameter } & \multirow{2}{*}{$\begin{array}{l}\text { All patients } \\
(n=789)\end{array}$} & \multicolumn{2}{|c|}{ Autoimmune disease (AD) } & \multicolumn{2}{|c|}{ No autoimmune disease (NAD) } \\
\hline & & $\begin{array}{l}\text { Immunosuppressed } \\
\text { (IS) } \\
\text { IS-AD }(n=37)\end{array}$ & $\begin{array}{l}\text { Not immunosuppressed } \\
\text { (NIS) } \\
\text { NIS-AD }(n=39)\end{array}$ & $\begin{array}{l}\text { Immunosuppressed } \\
\text { (IS) } \\
\text { IS-NAD }(n=26)\end{array}$ & $\begin{array}{l}\text { Not immunosuppressed } \\
\text { (NIS) } \\
\text { NIS-NAD }(n=687)\end{array}$ \\
\hline Age, years (median [IQR]) & $64(54-73)$ & $61(55-69)$ & $68(57-77)$ & $65(56-73)$ & $64(54-73)$ \\
\hline Men, $n(\%)$ & $569(72.1)$ & $17(45.9)$ & $22(56.4)$ & $20(76.9)^{*}$ & $509(74.1)^{* *}$ \\
\hline \multicolumn{6}{|l|}{ Comorbidities, $n(\%)$} \\
\hline Hypertension & $353(44.7)$ & $13(35.1)$ & $15(38.5)$ & $10(38.5)$ & $315(45.9)$ \\
\hline Diabetes & $159(20.2)$ & $3(8.1)$ & $5(12.8)$ & $6(23.1)$ & $145(21.1)$ \\
\hline Obesity $^{\mathrm{a}}$ & $287(36.4)$ & $9(24.3)$ & $15(38.5)$ & $9(34.6)$ & $254(37.0)$ \\
\hline Cardiovascular disease $^{\mathrm{b}}$ & $132(16.7)$ & $5(13.5)$ & $8(20.5)$ & $6(23.1)$ & $113(16.4)$ \\
\hline Chronic renal disease & $53(6.7)$ & $3(8.1)$ & $3(7.7)$ & $9(34.6)^{*}$ & $38(5.5)^{+}$ \\
\hline Chronic liver disease ${ }^{\mathrm{c}}$ & $53(6.7)$ & $2(5.4)$ & $2(5.1)$ & $4(15.4)$ & $45(6.6)$ \\
\hline Chronic lung disease $^{\mathrm{d}}$ & $122(15.5)$ & $8(21.6)$ & $8(20.5)$ & $8(30.8)$ & $98(14.3)^{\dagger}$ \\
\hline Active malignancy & $47(6.0)$ & $2(5.4)$ & $3(7.7)$ & $16(61.5)^{* *}$ & $26(3.8)^{4}$ \\
\hline Malignancy in remission & $77(9.8)$ & $2(5.4)$ & $9(23.1)^{*}$ & $7(26.9)^{*}$ & $60(8.7)^{\dagger}$ \\
\hline Organ transplant & $13(1.6)$ & $1(2.7)$ & $0(0.0)$ & $8(30.8)^{*}$ & $4(0.6)^{\ddagger}$ \\
\hline HIV infection & $9(1.1)$ & $0(0.0)$ & $0(0.0)$ & $1(3.8)$ & $8(1.2)$ \\
\hline Dementia $^{\mathrm{e}}$ & $28(3.5)$ & $1(2.7)$ & $3(7.7)$ & $0(0.0)$ & $24(3.5)$ \\
\hline CCI score, median (IQR) & $3(1-4)$ & $3(2-4)$ & $4(2-5)$ & $6(3-8) *$ & $2(1-4)^{* *}$ \\
\hline $\begin{array}{l}\text { Bacterial coinfection, } n \\
\quad(\%)\end{array}$ & $127(16.1)$ & $7(18.9)$ & $13(33.3)$ & $5(19.2)$ & $102(14.9)$ \\
\hline
\end{tabular}

$C C I$, Charlson Comorbidity Index; $I Q R$, interquartile range

${ }^{\text {a }}$ Defined as a body mass index $\geq 30 \mathrm{~kg} / \mathrm{m}^{2}$

${ }^{\mathrm{b}}$ Heart failure, myocardiopathy, ischemic heart disease, and moderate-severe valvular heart disease

${ }^{\mathrm{c}}$ Chronic hepatitis, cirrhosis, hepatic steatosis, non-alcoholic steatohepatitis

${ }^{\mathrm{d}}$ Chronic obstructive pulmonary disease, obstructive sleep apnea-hypopnea syndrome, asthma, and diffuse interstitial lung disease

${ }^{\mathrm{e}}$ Includes developmental disabilities

${ }^{*} p<0.05$ vs IS-AD; ${ }^{* *} p<0.0001$ vs IS-AD; ${ }^{\dagger} p<0.05$ vs IS-NAD; ${ }^{*} p<0.0001$ vs IS-NAD 
Table 2 Unadjusted and adjusted Cox regression models for time to severe acute respiratory distress syndrome (ARDS; the primary endpoint), death, and the composite endpoint of mechanical/non-invasive ventilation or death

\begin{tabular}{|c|c|c|c|c|c|c|}
\hline \multirow[t]{2}{*}{ Endpoint } & \multicolumn{2}{|l|}{ Unadjusted } & \multirow[t]{2}{*}{$p$ value } & \multicolumn{2}{|l|}{ Adjusted $^{\mathrm{a}}$} & \multirow[t]{2}{*}{$p$ value } \\
\hline & Hazard ratio & $95 \% \mathrm{CI}$ & & Hazard ratio & $95 \% \mathrm{CI}$ & \\
\hline \multicolumn{7}{|l|}{ Time to severe ARDS } \\
\hline IS-AD & 0.37 & $0.19-0.68$ & 0.002 & 0.42 & $0.23-0.80$ & 0.008 \\
\hline NIS-AD & 0.96 & $0.63-1.47$ & 0.854 & 0.96 & $0.63-1.47$ & 0.85 \\
\hline IS-NAD & 1.34 & $0.86-2.08$ & 0.191 & 1.14 & $0.72-1.81$ & 0.566 \\
\hline NIS-NAD (reference) & 1 & & & & & \\
\hline \multicolumn{7}{|l|}{ Time to death } \\
\hline IS-AD & 0.83 & $0.30-2.25$ & 0.708 & 0.82 & $0.30-2.28$ & 0.71 \\
\hline NIS-AD & 0.80 & $0.35-1.82$ & 0.594 & 0.55 & $0.24-1.28$ & 0.165 \\
\hline IS-NAD & 3.15 & $1.76-5.67$ & $<0.0001$ & 1.71 & $0.94-3.12$ & 0.081 \\
\hline NIS-NAD (reference) & 1 & & & & & \\
\hline \multicolumn{7}{|c|}{ Time to composite of mechanical/non-invasive ventilation or death } \\
\hline IS-AD & 0.61 & $0.31-1.18$ & 0.139 & 0.66 & $0.34-1.29$ & 0.23 \\
\hline NIS-AD & 1.12 & $0.69-1.83$ & 0.648 & 1.05 & $0.64-1.72$ & 0.86 \\
\hline IS-NAD & 1.46 & $0.88-2.43$ & 0.139 & 1.14 & $0.67-1.93$ & 0.62 \\
\hline NIS-NAD (reference) & 1 & & & & & \\
\hline
\end{tabular}

$A D$, autoimmune disease; $C I$, confidence interval; $I S$, immunosuppressed; $N A D$, no autoimmune disease; $N I S$, not immunosuppressed. Italics: differences are statistically significant; hence, $p<0.05$

${ }^{a}$ Adjusted for sex, obesity, and Charlson Comorbidity Index score

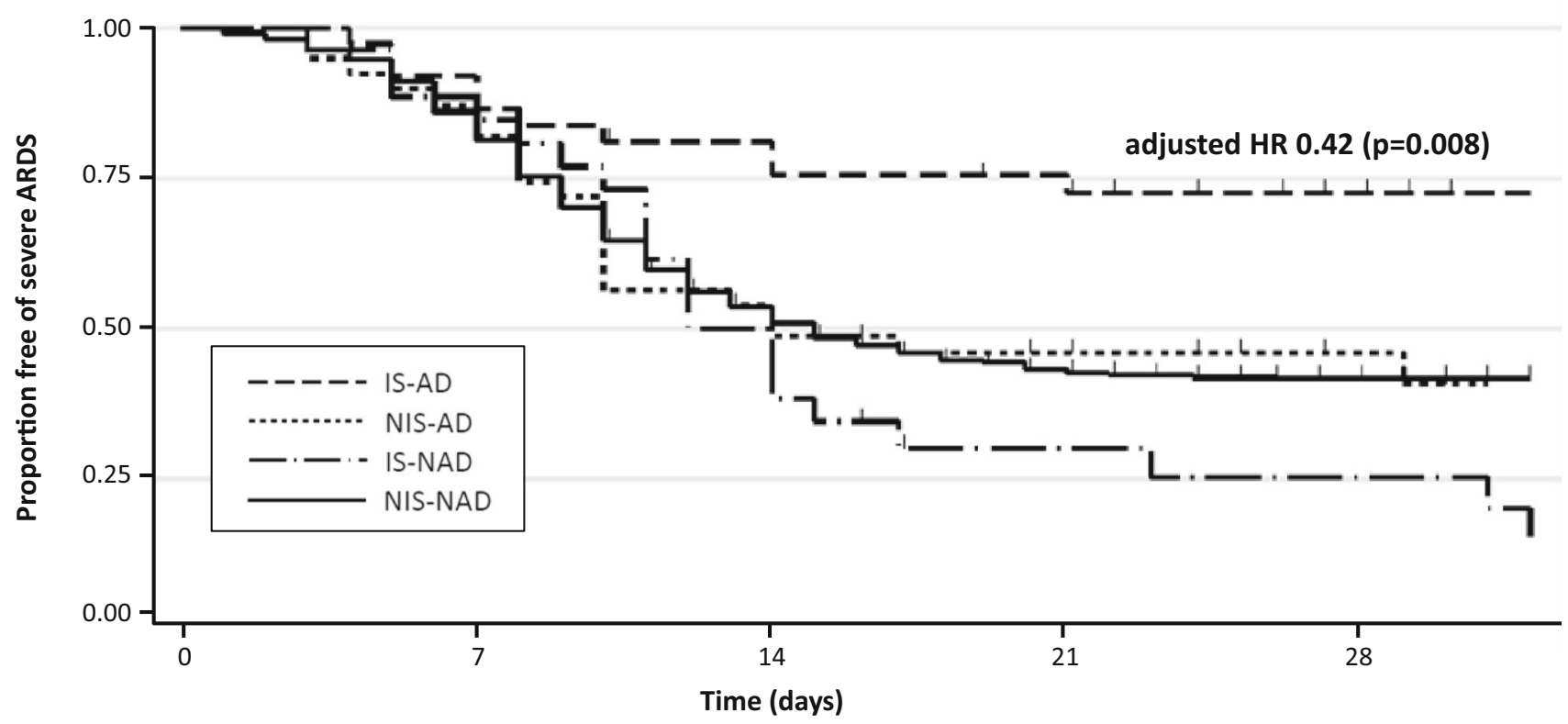

No. at risk:

$\begin{array}{lccc}\text { IS-AD } & 37 & 34 & 29 \\ \text { NIS-AD } & 39 & 34 & 21 \\ \text { IS-NAD } & 26 & 23 & 13 \\ \text { NIS-NAD (ref) } & 687 & 591 & 364\end{array}$

Fig. 2 Kaplan-Meier survival estimates for severe acute respiratory distress syndrome (ARDS) according to immunosuppression and autoimmune disease (AD) among 789 patients hospitalized with COVID-19.

$\begin{array}{ccc}29 & 26 & 18 \\ 21 & 16 & 9 \\ 13 & 6 & 5 \\ 364 & 276 & 218\end{array}$

COVID-19, coronavirus disease 2019; HR, hazard ratio; IS, immunosuppressed; NAD, no autoimmune disease; NIS, not immunosuppressed; ref, reference 
as time-dependent covariates; and (ii) according to whether or not immunosuppressive treatment was discontinued on admission. Both sensitivity analyses yielded similar results to the base-case (Supplementary Tables S4 and S5).

Serum levels of IL-6 were available for 789 patients. We excluded data from patients receiving either interferon- $\beta$ or high doses of corticosteroids ( $\geq 250 \mathrm{mg} /$ day methylprednisolone equivalent) prior to blood sampling $(n=136)$, leaving a sample size of 653 patients. The median time between onset of symptoms and IL-6 measurement for included patients was 11 (IQR 8-14) days, with no differences between cohorts. IL-6 levels were found to be significantly higher in the IS-NAD cohort compared with either the IS-AD or NIS-NAD cohorts (Fig. 3 and Supplementary Table S4). In contrast, there were no significant differences between any of the cohorts in serum levels of IL-1B, IL-8, IL-10, IL-12p70, or TNF- $\alpha$.

A final outcome (death or discharge) was observed in 603 (76.4\%) patients. A total of 124 patients $(20.6 \%)$ died between admission to HRC and 19 April 2020, with death occurring at a median of 16 (IQR 9-23) days after symptom onset and 13 (IQR 8-19) days after hospitalization. No patients died between discharge and the end of follow-up. We did not find any significant differences between the NIS-NAD and other cohorts in mortality rates; however, among those without an $\mathrm{AD}$, there was a trend towards an increased risk of death in immunosuppressed (IS-NAD) vs non-immunosuppressed (NIS-NAD) patients (adjusted HR 1.71; 95\% CI 0.94-3.12; $p=0.081$; Table 2). Before adjustment, the difference

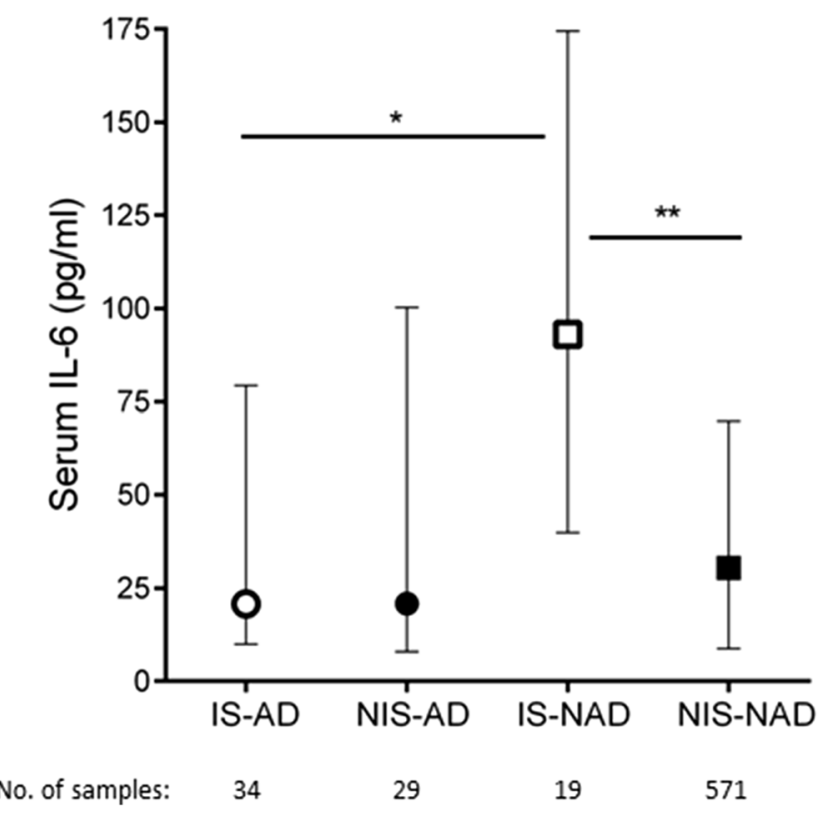

Fig. 3 Serum levels of interleukin (IL)-6 according to immunosuppression and autoimmune disease (AD). IS, immunosuppressed; NAD, no autoimmune disease; NIS, not immunosuppressed. For each cohort, the symbols represent the median value and the whiskers show the interquartile range. $* p=0.0328 ; * * p=0.0057$ between these two cohorts was highly statistically significant (HR 3.15; 95\% CI 1.76-5.67; $p<0.0001$ ).

The overall cumulative incidence of mortality at 14 and 28 days from symptom onset was $8.2 \%$ and $24.1 \%$, respectively (Supplementary Table S5). There were no significant differences in mortality at 14 and 28 days between patients with an $\mathrm{AD}$ (4.5\% and $23.4 \%$, respectively) and those with no AD $(8.6 \%$ and $24.2 \%$, respectively) (adjusted HR 0.60; $95 \%$ CI $0.31-1.16 ; p=0.13)$.

Overall, the composite endpoint of MV/NIV or death was observed in $9.4 \%$ and $46.4 \%$ of patients after 7 and 28 days, respectively (Supplementary Table S6). Results at these timepoints were similar in the $\mathrm{AD}(15.8 \%$ and $41.1 \%)$ and NAD (18.5\% and 58.8\%) cohorts (adjusted HR 0.86; 95\% CI $0.57-$ $1.30 ; p=0.48)$. We did not find any significant differences between the NIS-NAD and other cohorts on this endpoint (Table 2).

Several drugs were used to treat COVID-19 (Supplementary Table S7). Consistent with findings on serum IL-6 levels (Fig. 3 and Supplementary Table S3), patients in the IS-NAD cohort were significantly more likely than those in the IS-AD and NIS-NAD cohorts to receive tocilizumab; patients in the NIS-NAD cohort were more likely to receive lopinavir/ritonavir than those in the IS-NAD cohort. No other significant differences in COVID-19 treatments were found.

\section{Discussion}

Although most individuals with SARS-CoV-2 infection experience no or only mild clinical symptoms, a small proportion of patients will require hospitalization and progress to severe ARDS, multiple organ failure and death, sometimes accompanied by vasculitis and dysregulated coagulation [9]. COVID-19-related death results, in most cases, from respiratory failure, cardiac injury, or both [10]. Well-established predictors of this more severe clinical course include older age, male sex, and underlying comorbidities $[2,11,12]$.

Based on experience with respiratory viruses such as influenza [13], concerns were initially expressed that immunosuppressed individuals might be at increased risk of developing a more severe clinical illness. However, multiple studies have now shown that severe COVID-19 is caused by the development of a hyper-inflammatory state, characterized by dysregulation of the immune system and cytokine storm: the uncontrolled, excessive release of pro-inflammatory $\mathrm{T}_{\mathrm{h}} 1$-associated cytokines and chemokines, including interferon- $\gamma$, IL- $1 \beta$, IL6 , TNF- $\alpha$, monocyte chemoattractant protein-1 (MCP-1), and CXCL10 [9]. In this sense, COVID-19 has a similar underlying pathophysiology to SARS and Middle East respiratory syndrome (MERS) [9, 14, 15]. Thus, several immunomodulatory drugs are being investigated for the treatment of COVID-19 [9, 16]. 
Other evidence suggests that the pathologic hyperactivity of the innate immune system observed in severe COVID-19 may be caused by a deficit in the adaptive immune response caused in turn by immunosenescence, immunosuppression, or virus-induced lymphopenia [17]. This is suggested by the high levels of IL-6 and IL-10 that are seen in patients with severe disease [18]. The lack of an effective T cell response may hinder viral clearance, allowing a sustained, deleterious innate immune response to develop. This hypothesis is consistent with the observed associations between more severe disease, older age [11], and lymphopenia [18].

Thus, it is important to ascertain the impact of immunosuppression and autoimmunity on the clinical course of COVID19. In a series of 82 patients with confirmed or highly suspected COVID-19, all of whom had an AD, patients who did not require hospital treatment for COVID-19 were more likely to be on immunosuppressive therapy than those who were hospitalized (76\% vs 50\%) [19]. In an Italian study, 123 of 784 (15.7\%) patients with MS and suspected or confirmed COVID-19, 83\% whom were receiving disease-modifying therapy (DMT) for MS, had a severe course of infection [20]; this was a similar percentage to that reported in the general population [21]. In univariate analysis, the odds of severe COVID-19 were significantly higher in patients not receiving DMT (OR 2.83; $p<0.001$ ), but varied according to the specific drug used in DMT-treated patients. Thus, relative to dimethyl fumarate, a greater risk of severe COVID-19 illness was observed for anti-CD20 therapies (ocrelizumab and rituximab; OR 2.93; $p=0.003$ ), but not for other MS treatments such as interferon-beta, fingolimod, and natalizumab [20].

Several recent observational studies and case series support the hypothesis that immunosuppression, whether pathologic or therapeutic, may reduce the risk of severe COVID-19 illness. For example, although patient numbers are low, preliminary findings suggest that most patients with agammaglobulinemia $[22,23]$ and many of those with other inborn errors of immunity [24] have a mild course of COVID-19 disease, and do not require admission to intensive care or MV. Additionally, it has been suggested that treatment with a Bruton tyrosine kinase inhibitor may protect against severe respiratory disease in patients with a B cell malignancy and COVID-19 [25, 26].

Overall, these findings indicate that the relationship between immune function and COVID-19 outcomes is highly complex, and warrants further investigation.

In an initial study of 138 patients admitted to HRC with confirmed or highly suspected COVID-19, moderate or severe ARDS was significantly less likely to develop in (nonseverely) immunosuppressed compared with nonimmunosuppressed individuals ( $22.2 \%$ vs $49.5 \%$; $p=0.004)$. However, further analysis showed that, in this population of non-severely immunosuppressed individuals, only those with an $\mathrm{AD}$ had a reduced risk of moderate or severe ARDS (adjusted OR 0.12; $p=0.007$ ) [6].
Clearly, immunosuppressed individuals are not a homogeneous group. Many patients will have demographic characteristics or comorbidities that modify their risk for severe COVID-19 disease. For this reason, we undertook the present, more complex, study of consecutive admissions to HRC. As expected, we found that patients who were immunosuppressed and had an $\mathrm{AD}$ (the IS-AD cohort) had a significantly reduced risk of progressing to severe ARDS relative to those with neither an AD nor immunosuppression. However, no such reduction in risk was seen for immunosuppressed patients who did not have an AD. Indeed, there were proportionately more cases of severe ARDS, and more deaths, in this group than in the NIS-NAD cohort, even after adjusting for sex, obesity, and CCI score.

Most patients in the IS-NAD cohort had a history of malignant disease or were solid organ transplant recipients. In a recent large database study in the UK, malignant disease and solid organ transplantation were associated with significantly elevated risks of in-hospital death among patients with COVID-19 [27]. A possible explanation for our observation, therefore, is that the inherent lymphocyte activation present in $\mathrm{AD}$ counteracts the detrimental effect of mild-to-moderate immunosuppression, whereas this does not occur in other immunosuppressed patients. This idea is supported by recent reports of more severe COVID-19 disease and/or higher mortality among patients with immunosuppression related to hematologic malignancy [27-29] or solid organ transplantation $[27,30,31]$ compared to non-immunosuppressed individuals. However, not all studies of COVID-19 in solid organ transplant recipients have found unequivocal evidence of more severe disease and/or an increased risk of death in this population compared with controls $[32,33]$.

We found significantly higher levels of IL-6 in patients in the IS-NAD cohort, compared with either the IS-AD or NIS-NAD cohorts; elevated IL-6 levels have been associated with poor outcomes in patients with COVID-19 [18]. Consistent with this, patients in the IS-NAD cohort were more likely to be prescribed tocilizumab than those in other cohorts. Interestingly, however, we did not find significant differences between cohorts in any of the other cytokines measured. Our observations support previous findings suggesting that IL-6 up-regulation may derive from a suboptimal lymphocyte response [17], and show that adaptive immune cell activation may be beneficial in COVID-19, even if patients are receiving IS treatment. Furthermore, cytokine storm appears to be restricted in patients with COVID-19 to IL-6 production, although a localized increase in IL-1 $\beta$ in the lungs cannot be completely excluded.

There are obvious limitations in a single-center study of this type. First, we included only hospitalized patients, and this limits the generalizability of our findings. The requirement for cytokine level data would also have favored the inclusion of more severely ill patients. Second, although the sample size was large, there was significant imbalance 
between the cohorts. Three of the cohorts had fewer than 40 patients each, while the NIS-NAD cohort had over 600 . Third, the relatively short follow-up time means that some events of interest may have occurred after the cut-off date. Fourth, we selected severe ARDS as the endpoint of interest, but cytokine storm-which we suggest may be abrogated by immunosuppression - has other life-threatening sequelae, including multiple organ failure, cardiac injury, and renal impairment $[10,12,34,35]$. We did not specifically consider these outcomes. Lastly, standardized care pathways and evidence-based treatment protocols for COVID-19 have not yet been developed, and differences in the management of the disease could have affected outcomes or introduced bias.

Despite these limitations, we believe our findings could have direct relevance to physicians treating immunosuppressed patients with an AD. Suspension of effective immunosuppressive treatment in these patients may jeopardize AD disease control, with potentially serious consequences [36, 37]. Such action may not be necessary; however, careful, tailored decision-making, taking into account all of an individual's known risk factors, is critical. In contrast, our findings in non- $\mathrm{AD}$ immunosuppressed patients suggest that immunosuppression may increase the risk of severe COVID-19 outcomes in patients with cancer and recipients of a solid organ transplant.

In conclusion, among hospitalized patients with COVID19 , those with immunosuppression and an AD appear significantly less likely to progress to severe ARDS than patients with neither immunosuppression nor an AD. Further studies, utilizing a prospective design and a larger sample size would provide valuable additional information on the role of immunosuppression in COVID-19.

Supplementary Information The online version contains supplementary material available at https://doi.org/10.1007/s10875-020-00927-y.

Acknowledgments We would like to thank Richard Crampton of Springer Healthcare Communications who wrote the first and subsequent drafts of the manuscript. This medical writing assistance was funded by an independent grant from Merck S.L.U., Spain.

Author Contributions Drs. Monreal, Sainz de la Maza, and Villar had full access to all of the data in the study and take responsibility for the integrity of the data and the accuracy of the data analysis.

Study concept and design: Monreal, Sainz de la Maza, and Villar.

Acquisition, analysis, or interpretation of data: Monreal, Sainz de la Maza, Fernández-Velasco, Natera-Villalba, Rita, Rodríguez-Jorge, Beltrán-Corbellini, Iturrieta-Zuazo, Rodríguez de Santiago, Espiño, De Andrés, Fortún, Barbero, Vázquez, Fernández Lucas, Manzano, Montero-Errasquín, Costa-Frossard, Masjuan, and Villar.

Drafting of the manuscript: Monreal, Sainz de la Maza, Rodríguez de Santiago, and Villar.

Critical revision of the manuscript for important intellectual content: Monreal, Sainz de la Maza, Fernández-Velasco, Natera-Villalba, Rita, Rodríguez-Jorge, Beltrán-Corbellini, Iturrieta-Zuazo, Rodríguez de Santiago, Espiño, De Andrés, Fortún, Barbero, Vázquez, Fernández Lucas, Manzano, Montero-Errasquín, Costa-Frossard, Masjuan, and Villar.

Statistical analyses: Monreal and Rodríguez de Santiago.
Administrative, technical, or material support: Monreal, Sainz de la Maza, Fernández-Velasco, Natera-Villalba, Rita, Rodríguez-Jorge, Beltrán-Corbellini, Iturrieta-Zuazo, Rodríguez de Santiago, Espiño, De Andrés, and Villar.

Study supervision: Villar.

Funding No funding was received in connection with the design or conduct of the study, or the analysis or interpretation of the results. Funding was received for medical writing assistance (see below).

Data Availability The datasets generated during and/or analyzed during the current study are available from the corresponding author on reasonable request.

\section{Compliance with Ethical Standards}

Conflict of Interest The authors declare that they have no conflict of interest.

Ethical Approval The study was approved by the institutional ethics board of HRC. Due to its retrospective design, the need for informed consent from individual patients was waived.

\section{References}

1. World Health Organization. Novel coronavirus - China. 2020. www.who.int/csr/don/12-january-2020-novel-coronavirus-china/ en/. Accessed 22 May 20202020.

2. Huang C, Wang Y, Li X, Ren L, Zhao J, Hu Y, et al. Clinical features of patients infected with 2019 novel coronavirus in Wuhan, China. Lancet. 2020;395(10223):497-506. https://doi. org/10.1016/S0140-6736(20)30183-5.

3. Wu C, Chen X, Cai Y, Xia J, Zhou X, Xu S, et al. Risk factors associated with acute respiratory distress syndrome and death in patients with coronavirus disease 2019 pneumonia in Wuhan, China. JAMA Intern Med. 2020;180:934-43. https://doi.org/10. 1001/jamainternmed.2020.0994.

4. Siddiqi HK, Mehra MR. COVID-19 illness in native and immunosuppressed states: a clinical-therapeutic staging proposal. J Heart Lung Transplant. 2020;39(5):405-7. https://doi.org/10.1016/j. healun.2020.03.012.

5. D'Antiga L. Coronaviruses and immunosuppressed patients: the facts during the third epidemic. Liver Transpl. 2020;26(6):832-4. https://doi.org/10.1002/lt.25756.

6. Monreal E, de la Maza SS, Gullón P, Natera-Villalba E, ChicoGarcía JL, Beltrán-Corbellini Á et al. Non-severe immunosuppression might be associated with a lower risk of moderate-severe acute respiratory distress syndrome in COVID-19: a pilot study. J Med Virol. 2020. https://doi.org/10.1002/jmv.26656.

7. Ards Definition Task Force, Ranieri VM, Rubenfeld GD, Thompson BT, Ferguson ND, Caldwell E, et al. Acute respiratory distress syndrome: the Berlin Definition. JAMA. 2012;307(23): 2526-33. https://doi.org/10.1001/jama.2012.5669.

8. Charlson ME, Pompei P, Ales KL, MacKenzie CR. A new method of classifying prognostic comorbidity in longitudinal studies: development and validation. J Chronic Dis. 1987;40(5):373-83. https://doi.org/10.1016/0021-9681(87)90171-8.

9. Zhang W, Zhao Y, Zhang F, Wang Q, Li T, Liu Z, et al. The use of anti-inflammatory drugs in the treatment of people with severe coronavirus disease 2019 (COVID-19): the perspectives of clinical immunologists from China. Clin Immunol. 2020;214:108393. https://doi.org/10.1016/j.clim.2020.108393. 
10. Ruan Q, Yang K, Wang W, Jiang L, Song J. Clinical predictors of mortality due to COVID-19 based on an analysis of data of 150 patients from Wuhan, China. Intensive Care Med. 2020;46(5):8468. https://doi.org/10.1007/s00134-020-05991-x.

11. Zhou F, Yu T, Du R, Fan G, Liu Y, Liu Z, et al. Clinical course and risk factors for mortality of adult inpatients with COVID-19 in Wuhan, China: a retrospective cohort study. Lancet. 2020;395(10229):1054-62. https://doi.org/10.1016/S01406736(20)30566-3.

12. Wang $\mathrm{D}, \mathrm{Hu} \mathrm{B}, \mathrm{Hu} \mathrm{C}, \mathrm{Zhu} \mathrm{F}$, Liu X, Zhang J, et al. Clinical characteristics of 138 hospitalized patients with 2019 novel coronavirus-infected pneumonia in Wuhan, China. JAMA. 2020;323:1061-9. https://doi.org/10.1001/jama.2020.1585.

13. Memoli MJ, Athota R, Reed S, Czajkowski L, Bristol T, Proudfoot $\mathrm{K}$, et al. The natural history of influenza infection in the severely immunocompromised vs nonimmunocompromised hosts. Clin Infect Dis. 2014;58(2):214-24. https://doi.org/10.1093/cid/cit725.

14. Hui DS, Azhar EI, Kim YJ, Memish ZA, Oh MD, Zumla A. Middle East respiratory syndrome coronavirus: risk factors and determinants of primary, household, and nosocomial transmission. Lancet Infect Dis. 2018;18(8):e217-e27. https://doi.org/10.1016/S14733099(18)30127-0.

15. Wong CK, Lam CW, Wu AK, Ip WK, Lee NL, Chan IH, et al. Plasma inflammatory cytokines and chemokines in severe acute respiratory syndrome. Clin Exp Immunol. 2004;136(1):95-103. https://doi.org/10.1111/j.1365-2249.2004.02415.x.

16. Mehta P, McAuley DF, Brown M, Sanchez E, Tattersall RS, Manson JJ, et al. COVID-19: consider cytokine storm syndromes and immunosuppression. Lancet. 2020;395(10229):1033-4. https://doi.org/10.1016/S0140-6736(20)30628-0.

17. Vardhana SA, Wolchok JD. The many faces of the anti-COVID immune response. J Exp Med. 2020;217(6):e20200678. https://doi. org/10.1084/jem.20200678.

18. Zhang J, Yu M, Tong S, Liu LY, Tang LV. Predictive factors for disease progression in hospitalized patients with coronavirus disease 2019 in Wuhan, China. J Clin Virol. 2020;127:104392. https:// doi.org/10.1016/j.jcv.2020.104392.

19. Haberman R, Axelrad J, Chen A, Castillo R, Yan D, Izmirly P, et al. Covid-19 in immune-mediated inflammatory diseases - case series from New York. N Engl J Med. 2020;383:85-8. https://doi.org/10. 1056/NEJMc2009567.

20. Sormani MP, De Rossi N, Schiavetti I, Carmisciani L, Cordioli C, Moiola L, et al. Disease modifying therapies and Covid-19 severity in multiple sclerosis. Lancet. 2020. https://doi.org/10.2139/ssrn. 3631244 .

21. World Health Organization. Clinical management of COVID-19. Interim guidance. 2020. https://www.who.int/publications/i/item/ clinical-management-of-covid-19. Accessed 22 Oct 2020.

22. Quinti I, Lougaris V, Milito C, Cinetto F, Pecoraro A, Mezzaroma I, et al. A possible role for B cells in COVID-19? Lesson from patients with agammaglobulinemia. J Allergy Clin Immunol. 2020;146(1):211-3 e4. https://doi.org/10.1016/j.jaci.2020.04.013.

23. Soresina A, Moratto D, Chiarini M, Paolillo C, Baresi G, Foca E, et al. Two X-linked agammaglobulinemia patients develop pneumonia as COVID-19 manifestation but recover. Pediatr Allergy Immunol. 2020;31(5):565-9. https://doi.org/10.1111/pai.13263.

24. Meyts I, Bucciol G, Quinti I, Neven B, Fischer A, Seoane E, et al. Coronavirus disease 2019 in patients with inborn errors of immunity: an international study. J Allergy Clin Immunol. 2020. https://doi.org/10.1016/j.jaci.2020.09.010.

25. Thibaud S, Tremblay D, Bhalla S, Zimmerman B, Sigel K, Gabrilove J. Protective role of Bruton tyrosine kinase inhibitors in patients with chronic lymphocytic leukaemia and COVID-19. Br J Haematol. 2020;190(2):e73-e6. https://doi.org/10.1111/bjh.16863.

26. Treon SP, Castillo JJ, Skarbnik AP, Soumerai JD, Ghobrial IM, Guerrera ML, et al. The BTK inhibitor ibrutinib may protect against pulmonary injury in COVID-19-infected patients. Blood. 2020;135(21):1912-5. https://doi.org/10.1182/blood.2020006288.

27. Williamson E, Walker AJ, Bhaskaran KJ, Bacon S, Bates C, Morton CE, et al. OpenSAFELY: factors associated with COVID-19-related hospital death in the linked electronic health records of 17 million adult NHS patients. MedRxIV. 2020. https://doi.org/10.1101/2020.05.06.20092999.

28. He W, Chen L, Chen L, Yuan G, Fang Y, Chen W, et al. COVID19 in persons with haematological cancers. Leukemia. 2020;34(6): 1637-45. https://doi.org/10.1038/s41375-020-0836-7.

29. Martin-Moro F, Marquet J, Piris M, Michael BM, Saez AJ, Corona $\mathrm{M}$, et al. Survival study of hospitalised patients with concurrent COVID-19 and haematological malignancies. Br J Haematol. 2020;190:e16-20. https://doi.org/10.1111/bjh.16801.

30. Pereira MR, Mohan S, Cohen DJ, Husain SA, Dube GK, Ratner LE, et al. COVID-19 in solid organ transplant recipients: initial report from the US epicenter. Am J Transplant. 2020;20:1800-8. https://doi.org/10.1111/ajt.15941.

31. Akalin E, Azzi Y, Bartash R, Seethamraju H, Parides M, Hemmige $\mathrm{V}$, et al. Covid-19 and kidney transplantation. N Engl J Med. 2020;382:2475-7. https://doi.org/10.1056/NEJMc2011117.

32. Rinaldi M, Bartoletti M, Bussini L, Pancaldi L, Pascale R, Comai G, et al. COVID-19 in solid organ transplant recipients: no difference in survival compared to general population. Transpl Infect Dis. 2020:e13421. https://doi.org/10.1111/tid.13421.

33. Sharma P, Chen V, Fung CM, Troost JP, Patel VN, Combs M, et al. COVID-19 outcomes among solid organ transplant recipients: a case-control study. Transplantation. 2020. https://doi.org/10.1097/ TP.0000000000003447.

34. Guo T, Fan Y, Chen M, Wu X, Zhang L, He T, et al. Cardiovascular implications of fatal outcomes of patients with coronavirus disease 2019 (COVID-19). JAMA Cardiol. 2020;5:811-8. https://doi.org/ 10.1001/jamacardio.2020.1017.

35. Chen T, Wu D, Chen H, Yan W, Yang D, Chen G, et al. Clinical characteristics of 113 deceased patients with coronavirus disease 2019: retrospective study. BMJ. 2020;368:m1091. https://doi.org/ 10.1136/bmj.m1091.

36. Wolf D, Skup M, Yang H, Fang AP, Kageleiry A, Chao J, et al. Clinical outcomes associated with switching or discontinuation from anti-TNF inhibitors for nonmedical reasons. Clin Ther. 2017;39(4):849-62 e6. https://doi.org/10.1016/j.clinthera.2017.03. 005 .

37. Barry B, Erwin AA, Stevens J, Tornatore C. Fingolimod rebound: a review of the clinical experience and management considerations. Neurol Ther. 2019;8(2):241-50. https://doi.org/10.1007/s40120019-00160-9.

Publisher's Note Springer Nature remains neutral with regard to jurisdictional claims in published maps and institutional affiliations. 